\title{
Research Article \\ On the Accuracy of Fluid Approximations to a Class of Inventory-Level-Dependent EOQ and EPQ Models
}

\author{
Alexey Piunovskiy and Yi Zhang \\ Department of Mathematical Sciences, University of Liverpool, Liverpool, L69 7ZL, UK \\ Correspondence should be addressed to Alexey Piunovskiy, piunov@liv.ac.uk
}

Received 29 September 2010; Accepted 18 January 2011

Academic Editor: Viliam Makis

Copyright (C) 2011 A. Piunovskiy and Y. Zhang. This is an open access article distributed under the Creative Commons Attribution License, which permits unrestricted use, distribution, and reproduction in any medium, provided the original work is properly cited.

\begin{abstract}
Deterministic Economic Order Quantity (EOQ) models have been studied intensively in the literature, where the demand process is described by an ordinary differential equation, and the objective is to obtain an EOQ, which minimizes the total cost per unit time. The total cost per unit time consists of a "discrete" part, the setup cost, which is incurred at the time of ordering, and a "continuous" part, the holding cost, which is continuously accumulated over time. Quite formally, such deterministic EOQ models can be viewed as fluid approximations to the corresponding stochastic EOQ models, where the demand process is taken as a stochastic jump process. Suppose now an EOQ is obtained from a deterministic model. The question is how well does this quantity work in the corresponding stochastic model. In the present paper we justify a translation of EOQs obtained from deterministic models, under which the resulting order quantities are asymptotically optimal for the stochastic models, by showing that the difference between the performance measures and the optimal values converges to zero with respect to a scaling parameter. Moreover, we provide an estimate for the rate of convergence. The same issue regarding specific Economic Production Quantity (EPQ) models is studied, too.
\end{abstract}

\section{Introduction}

Consider an inventory item which is demanded. So the inventory level gradually decreases and is backed up by ordering new inventories from time to time. There are two costs to consider: a positive inventory level results in a holding cost, and every order induces a setup cost. The objective is to determine an order quantity that minimizes the total cost per unit time (tcu). Such a minimizer is known as an Economic Order Quantity (EOQ), and the model itself is known as an EOQ model. 
Arguably the simplest EOQ model (sometimes referred to as the classic EOQ model) is based on the following assumptions: (i) the instantaneous holding cost rate is constant; (ii) the setup cost is constant; (iii) the demand comes in a deterministic and continuous process at a constant rate; (iv) no backlogging is allowed so that at inventory level zero all the arrived demand is rejected; $(\mathrm{v})$ the inventory item is homogeneous and nonperishable so that only the demand reduces the inventory level; (vi) the inventory level is reviewed continuously so that it can be described by an ordinary differential equation; (vii) the replenishment takes place instantaneously after ordering. A rigorous description of the classic EOQ model is shortly given in Section 2.1. Amongst the efforts of generalizing the classic EOQ model, a great deal have been made on relaxing assumption (iii). For instance, the EOQ model considered in [1] (see also the references therein) assumes the demand rate to be inventory level dependent, despite the dependence is of a specific form. This is a response to: "At times, the presence of inventory has a motivational effect on the people around it. It is a common belief that large piles of goods displayed in a supermarket will lead the customer to buy more [2]." Generalizations regarding other assumptions include allowing backlogging and periodic reviews in [3], accounting for perishable goods in [4-7], and so on. A comprehensive review of the literature on EOQ models is available in [8]. Note that the aforementioned works mainly focus on EOQ models where the demand process is deterministic. Quite formally these deterministic models can be viewed as the fluid approximations to the corresponding stochastic EOQ models, where the demand comes in a stochastic jump process. (For this reason, in what follows we call the deterministic models also fluid models, and EOQs derived from fluid models are referred to as fluid EOQs.)

Suppose now that a fluid EOQ is obtained and the corresponding stochastic EOQ model is appropriately formulated. Then the issue of interest is how to translate the fluid EOQ into an order quantity for the stochastic model, where the expected total cost per unit time (TCU) is (nearly) minimal. The fluid approximation would be justified if such a translation is obtained. The formal justification of the fluid approximations to various jump Markov optimization models with local transitions has been addressed by numerous authors, see [9-12], all of which focus on queueing networks. In greater detail the optimization problems considered in $[9,12]$ are with a discounted criterion over an infinite horizon, the one in [11] is with an expected total cost criterion over a fixed finite time horizon, while the performance measure for the queueing network in [10] is the expected total cost up to the first moment the system gets empty. Here we emphasize that in fluid (resp., stochastic) EOQ models the performance measures are tcus (resp., TCUs), which are long run averages. All of those works compare the optimal value for the fluid model with the performance measure for the (scaled) stochastic model under the translated policy and show that the difference converges to zero as the scaling parameter increases to infinity. However, none of them reveal the rate of convergence, which measures the accuracy of the fluid approximations and the efficiency of the underlying translations. Consequently the more recent development on this topic aims at obtaining the rate of convergence, see [13-15]. In particular, in [14] the author proposes a translation of the fluid EOQ and shows that it results in an asymptotically fluid optimal (AFO) and asymptotically optimal (AO) order quantity for the corresponding stochastic model. (The accurate definitions of "AFO" and "AO" are postponed to Sections 2 and 3.) However, fairly strong conditions on the system parameters are assumed there, restricting the applicability of the obtained results.

Therefore, the main contribution of this paper is to provide a refinement of the results obtained in [14] by relaxing the conditions assumed therein, and thus enlarging the applicability to cover a broader class of EOQ models. In greater detail, by taking the stochastic 
model as a continuous-time Markov chain, we justify the translation of fluid EOQ proposed in [14] and obtain its efficiency in the form of a rate of convergence. The Markov property of our stochastic model is a result of assuming exponentially distributed interarrival times in the demand process, which is standard in the current literature on inventory systems, see $[7,16-19]$. In our models, the demand and holding cost rates are of a rather general inventory-level dependence, and thus with broad applicabilities. In particular, our results are applicable to the important case of discontinuous demand and holding cost rates (compared to that globally Lipschitz continuous rates are assumed in [14]), see more discussions on this in Section 4. Moreover, in this paper results similar to those for EOQ models are derived for Economic Production Quantity (EPQ) models, too.

The rest of this paper is organized as follows. In Sections 2 and 3 we formulate EOQ and EPQ models and state the main results. In Section 4 some comments are given on the issues of possible applicabilities of the obtained results and thus illustrate the contribution of this paper. We finish this paper with conclusions. The proofs of the main statements are postponed to the appendix.

\section{Economic Order Quantity Models}

In what follows, the trivial case of an order quantity taking zero is excluded from consideration, and the context should always make it clear when [.] stands for the function taking the largest integer part of its argument.

\subsection{Description of Mathematical Models}

\section{(a) Fluid Model}

Suppose some order quantity $y>0$ is fixed. Then let $\{x(t), t \geq 0\}$ be the inventory level process subject to the dynamics $d x / d t=-\mu(x)$ when $0<x(t) \leq y$ and $x\left(t_{\text {order }}+0\right)=y$, where $x(t)$ reaches state zero at $t_{\text {order }}$. Here, $\mu(x)>0$ is the demand rate, and the impulsive jump of $x(t)$ at $t_{\text {order }}$ reflects the instantaneous replenishment assumption. Let $t_{\text {cycle }}$ be the time duration between two consecutive jumps of the inventory level process, $g(x)$ the instantaneous holding cost rate, and $K>0$ the setup cost, incurred immediately whenever an order is made. We are interested in minimizing the long run average cost given by

$$
\mathrm{tcu}(y) \triangleq \lim _{T \rightarrow \infty} \frac{1}{T}\left\{\int_{0}^{T} g(x(t)) d t+K\left[\frac{T}{t_{\text {cycle }}}\right]\right\}
$$

Let us call $y^{*}$ the EOQ for the fluid model, so that $\operatorname{tcu}\left(y^{*}\right)=\inf _{y>0} \operatorname{tcu}(y)$. In particular, if $\mu(x)=\mu>0$ and $g(x)=h x$ (i.e., the demand rate and the holding cost rate per unit of inventory are constants $\mu>0, h>0)$, then we have the so called classic EOQ model.

\section{(b) (Scaled) Stochastic Model}

In the corresponding stochastic model, the inventory item is measured in small units, so that a scaling parameter $n=1,2, \ldots$ is present to indicate the units. The intuitive meaning of this scaling parameter is explained in Remark 2.1 below. In greater detail, fixing some integer 
order quantity ${ }^{n} Y>0$, the inventory level process $\left\{{ }^{n} X_{t}, t \geq 0\right\}$ is modelled as a continuoustime Markov chain with the state space $\left\{0,1,2, \ldots,{ }^{n} Y\right\}$ and the transition rates $\left\{{ }^{n} A_{i, j}\right\}_{i, j=0}^{n^{n}}$ given by

$$
{ }^{n} A_{0, j}= \begin{cases}n \mathcal{K}, & \text { if } j={ }^{n} \Upsilon \\ -n \kappa, & \text { if } j=0 \\ 0, & \text { otherwise }\end{cases}
$$

and in the case of $i \in\left\{1,2,3, \ldots,{ }^{n} Y\right\}$

$$
{ }^{n} A_{i, j}= \begin{cases}n \mu\left(\frac{i}{n}\right), & \text { if } j=i-1 \\ -n \mu\left(\frac{i}{n}\right), & \text { if } j=i \\ 0, & \text { otherwise }\end{cases}
$$

where $n \mu(i / n)$ is the instantaneous demand rate and $n \kappa$ is the parameter of the exponentially distributed lead time between the ordering and the corresponding replenishment. In other words, the time between two consecutive demand arrivals is exponentially distributed with mean $1 / n \mu(i / n)$ when the current inventory level is $i$, and new inventories are ordered when the inventory level hits zero.

Remark 2.1. The scaled stochastic model can be linked to the fluid model by taking ${ }^{n} Y \triangleq[n y]$, where $y>0$ is the order quantity for the fluid model (Here $[n y]>0$ for big enugh $n$. So below we assume that $[n y]>0$, that is, we consider large enough $n$.). The above-described scaling is often referred to as a fluid scaling, and its intuitive meaning can be understood as follows. Clearly, when $n=1$, the stochastic model is a corresponding version of the fluid model. As we increase $n$, the demand comes in smaller units, and inventories are measured more accurately. Take $\mu(i / n) \triangleq M$ as an example. Suppose that $n=1$ corresponds to the unit of $(\mathrm{kg})$ so that on average $1 \cdot \mu(i / 1)=M(\mathrm{~kg})$ units of demand come per time unit. In the case of $n=1000$, the unit will be $(\mathrm{g})$ : on average $n \mu(i / n)=1000 M(\mathrm{~g})$ units of demand come per time unit. Meanwhile, the cost rate is not amplified by multiplying $n$ because it costs the same to hold either $1000 M(\mathrm{~g})$ or $M(\mathrm{~kg})$ of inventories.

We are interested in minimizing the performance measure given by

$$
{ }^{n} \mathrm{TCU}\left({ }^{n} Y\right) \triangleq \lim _{T \rightarrow \infty} \frac{1}{T} E_{n}\left[\int_{0}^{\infty}\left\{g\left(\frac{{ }^{n} X_{t}}{n}\right)+K n \mu\left(\frac{1}{n}\right) I\left\{{ }^{n} X_{t}=1\right\}\right\} d t\right],
$$

where $E_{n} Y$ denotes the expectation operator with the initial inventory level (immediately after the replenishment) ${ }^{n} X_{0}={ }^{n} Y$. Let us call ${ }^{n} Y^{*}$ the EOQ for the (scaled) stochastic model, so that ${ }^{n} \mathrm{TCU}\left({ }^{n} Y^{*}\right)=\inf _{n} Y=1,2, \ldots{ }^{n} \mathrm{TCU}\left({ }^{n} Y\right)$.

We say that order quantity $[n y]$ for the stochastic model is AFO if

$$
\left.\lim _{n \rightarrow \infty}\right|^{n} \operatorname{TCU}([n y])-\operatorname{tcu}\left(y^{*}\right) \mid=0
$$


and $\mathrm{AO}$ if

$$
\lim _{n \rightarrow \infty}\left|{ }^{n} \mathrm{TCU}([n y])-{ }^{n} \mathrm{TCU}\left({ }^{n} Y^{*}\right)\right|=0
$$

In what follows, the EOQs for both fluid and stochastic models are assumed to be unique, and the similar assumption applies to the EPQ models.

\subsection{Main Results}

Condition 1. (a) There exist constants $d_{1} \geq 0, k_{1}>0$ and $\delta>0$ such that $\delta \leq \mu(x) \leq k_{1}$ and $|g(x)| \leq d_{1}$; here functions $\mu(\cdot)$ and $g(\cdot)$ are measurable and both defined on $[0, \infty)$.

(b) There exist finite intervals $\left(0, x_{1}\right),\left(x_{1}, x_{2}\right), \ldots$ with $\lim _{j \rightarrow \infty} x_{j}=\infty$ such that on each of them $g(x)$ and $\mu(x)$ are Lipschitz continuous with a common Lipschitz constant $d_{g}$ and $d_{\mu}$, respectively.

Note that Condition 1 implies that for any fixed $y>0$, there exist $L$ (possibly $y$ dependent) finite intervals $\left(0, x_{1}\right),\left(x_{1}, x_{2}\right), \ldots,\left(x_{L}, 3[y+1]\right)$ such that on each interval $1 / \mu(x)$ and $g(x) / \mu(x)$ are Lipschitz continuous with Lipschitz constants $d_{\mu} / \delta^{2}$ and $\left(d_{1} d_{\mu}+k_{1} d_{g}\right) / \delta^{2}$, respectively. For simplicity, we define $d_{2} \triangleq \max \left\{d_{\mu} / \delta^{2},\left(d_{1} d_{\mu}+k_{1} d_{g}\right) / \delta^{2}\right\}$.

Proposition 2.2. Under Condition 1, for any fixed order quantity in the fluid model $y>0$

$$
\begin{aligned}
& \left|{ }^{n} \operatorname{TCU}([n y])-\operatorname{tcu}(y)\right| \\
& \quad \leq \frac{\left(1+d_{1}\right) k_{1}^{2} \mathcal{\kappa}}{\delta\left([n y] \kappa+k_{1}\right)}\left\{\frac{k_{1} d_{2} 3[y+1]}{\delta}+\left(1+\frac{k_{1}}{\delta}\right) \frac{3 d_{1} L}{\delta}+\max \left\{\frac{g(0)}{\kappa}, \frac{1}{\mathcal{\kappa}}\right\}\right\} .
\end{aligned}
$$

In particular, Proposition 2.2 implies that $\left.\lim _{n \rightarrow \infty}\right|^{n} \operatorname{TCU}\left(\left[n y^{*}\right]\right)-\operatorname{tcu}\left(y^{*}\right) \mid=0$, that is, $\left[n y^{*}\right]$ is AFO.

The same calculations as in [14, page 406] result in the next lemma.

Lemma 2.3. Suppose that Condition 1 is satisfied and $g(x) \geq 0$, then

$$
y^{*} \geq \frac{\delta^{2} K}{k_{1} d_{1}}
$$

and ${ }^{n} Y^{*}$ satisfies ${ }^{n} Y^{*} / n \geq \delta^{2} K / k_{1} d_{1}-\delta / n \kappa$. In particular, $\underline{\lim }_{n \rightarrow \infty}\left({ }^{n} Y^{*} / n\right)>0$.

Corollary 2.4. Under Condition 1, if $\underline{\lim }_{n \rightarrow \infty}\left({ }^{n} Y^{*} / n\right)>0$ (see Lemma 2.3), then one has $\lim _{n \rightarrow \infty}\left|{ }^{n} Y^{*} / n-\left[n y^{*}\right] / n\right|=0$, and $\left.\lim _{n \rightarrow \infty}\right|^{n} \operatorname{TCU}\left(\left[n y^{*}\right]\right)-{ }^{n} \operatorname{TCU}\left({ }^{n} Y^{*}\right) \mid=0$, that is, $\left[n y^{*}\right]$ is $A O$.

Corollary 2.5. Under Condition 1, suppose in addition that $L$ is $y$-independent and $g(x) \geq 0$ on $[0, \infty)$. Then the following statements hold. 
(a) For any $y \geq \delta^{2} K / k_{1} d_{1}-\delta / n \kappa$ (see (2.8)) and any large enough $n \geq N$, where $N$ is a positive integer number satisfying $N>(2+\delta / \kappa) k_{1} d_{1} / \delta^{2} K$,

$$
\begin{aligned}
\left.\right|^{n} \operatorname{TCU} & ([n y])-\operatorname{tcu}(y) \mid \\
\leq & \frac{3\left(1+d_{1}\right) k_{1}^{3} d_{2}}{\delta^{2}} \frac{1}{n\left\{1-\kappa k_{1} d_{1} /\left(n \kappa \delta^{2} K-\delta k_{1} d_{1}\right)\right\}}+\frac{\left(1+d_{1}\right) k_{1}^{2}}{n \delta\left\{\delta^{2} K / k_{1} d_{1}-\delta / n \kappa-1 / n\right\}} \\
& \times\left\{\frac{3 k_{1} d_{2}}{\delta}+\left(1+\frac{k_{1}}{\delta}\right) \frac{3 d_{1} L}{\delta}+\max \left\{\frac{g(0)}{\kappa}, \frac{1}{\mathcal{\kappa}}\right\}\right\} \triangleq E(n) .
\end{aligned}
$$

Here the $y$-independent $E(n)$ goes to zero as fast as $1 / n$ in the sense of $E(n)=O(1 / n)$, whose meaning is that $\lim _{n \rightarrow \infty}(E(n) /(1 / n))=C$ with some (nonnegative) constant $C$.

(b) For any large enough $n \geq N$,

$$
\left|{ }^{n} \operatorname{TCU}\left({ }^{n} Y^{*}\right)-{ }^{n} \operatorname{TCU}\left(\left[n y^{*}\right]\right)\right| \leq 2 E(n)=O\left(\frac{1}{n}\right) .
$$

Here we recall that ${ }^{n} Y^{*}$ and $y^{*}$ are the EOQ for the (scaled) stochastic model and fluid model, respectively.

Corollary 2.5 refines Corollary 2.4 for certain cases by providing an estimate for the rate of convergence.

Fix some order quantity for the fluid model $y$ and scaling parameter $n$, and let ${ }^{n} \Pi(i), i=0,1, \ldots,[n y]$ be the stationary distribution of the inventory process $\left\{{ }^{n} X_{t}, t \geq 0\right\}$ and $\pi(x)$ the invariant density in the fluid model of the underlying dynamics of $x(t)$. Then the following proposition shows that the fluid model can also be used to provide approximations to stationary distributions of the inventory level process in the (scaled) stochastic model.

Proposition 2.6. Suppose that Condition 1 is satisfied and some order quantity for the fluid model $y>0$ is fixed. Then one has

$$
\left|{ }^{n} \Pi(0)-\pi(0)\right| \leq \frac{k_{1}}{k_{1}+\kappa[n y]}
$$

and for $i=1,2, \ldots,[n y]$

$$
\left|{ }^{n} \Pi(i)-\int_{(i-1) / n}^{i / n} \pi(x) d x\right| \leq \frac{2 y k_{1}^{2} \kappa / \delta^{2}+\left(k_{1}^{2} \kappa / n \delta\right)\left(3 k_{1} d_{2}(y+1) / \delta+\left(1+k_{1} / \delta\right) 3 d_{1} L / \delta+1 / \kappa\right)}{([n y]+1) y} .
$$

\subsection{A Comparison with Section 4 of [14]}

The fluid approximations of the EOQ models are also briefly considered in [14, Section 4]. Therefore, we mention the main difference of this paper from that one in this subsection.

Firstly, the present paper is based on a weaker condition. Indeed, instead of Condition 1, the following stronger condition is assumed in [14]. 
Condition 2. (a) Condition 1(a) holds.

(b) Functions $g(\cdot)$ and $\mu(\cdot)$ are globally Lipschitz continuous.

The global Lipschitz property is essential to the corresponding proof in [14].

Secondly, the approach in [14] is based on the explicit expression of ${ }^{n} \mathrm{TCU}\left({ }^{n} Y\right)$ obtained by solving the associated Poisson equations. Instead of doing that, the present paper employs the recent results of [15]. One advantage of this approach lies in the weaker condition required (only piecewise Lipschitz continuity is needed). Another advantage is that it allows one to study EPQ models, because the Poisson equations for EPQ models are much more difficult to solve compared to those for EOQ models. That is why EPQ models are not considered in [14].

\section{Economic Production Quantity Models}

In EOQ models the inventory is backed up at once by ordering new inventory items from external suppliers. In this section we consider the situation where the inventory is gradually backed up by producing new items. In greater detail, the inventory level decreases gradually to meet the demand, and when it hits zero, the production is switched on and new inventory items are being produced to back up the inventory. The production is switched off as soon as the inventory is backed up to a predetermined level. Here we have to account for the cost incurred from switching on the production as well as from holding the inventory items. The aim is to obtain an Economic Inventory Backup Level (EIBL) that minimizes TCU, and the resulting model is called an EPQ model. Similar to the previous section, below we justify the fluid approximations to stochastic EPQ models, whose rigorous description is shortly given.

\subsection{Description of Mathematical Models}

\section{(a) Fluid Model}

Suppose that we fix some real inventory backup level $y>0$, meaning that the production is always on until the inventory reaches the level $y$. Let $\{x(t), t \geq 0\}$ represent the inventory level process in the fluid model with state space $[0, y]$, and instantaneous demand and production rates $\mu(x)>0$ and $\lambda(x)>0$, respectively. Then the inventory level process is subject to the dynamics

$$
\frac{d x}{d t}= \begin{cases}-\mu(x) & \text { (production-off phase) } \\ \lambda(x)-\mu(x) & \text { (production-on phase) }\end{cases}
$$

where the production-off phase and production-on phase, superseding each other, are triggered by $x(t)=y$ and $x(t)=0$, respectively. In words, without any delay, once the inventory level reaches zero, production is switched on till it reaches the inventory backup level $y$. Let $g(x)$ be the holding cost rate, $K>0$ the setup cost incurred with switching on the 
production, and $t_{\text {cycle }}$ the time duration between two consecutive production switching-offs. So

$$
\mathrm{tcu}(y) \triangleq \lim _{T \rightarrow \infty} \frac{1}{T}\left\{\int_{0}^{T} g(x(t)) d t+K\left[\frac{T}{t_{\text {cycle }}}\right]\right\}
$$

Let us denote by $y^{*}$ the EIBL for the fluid model, so that tcu $\left(y^{*}\right)=\inf _{y>0} \operatorname{tcu}(y)$.

\section{(b) (Scaled) Stochastic Model}

Suppose that we fix some inventory backup level positive integer ${ }^{n} Y$, meaning that the production is always on until the inventory reaches level ${ }^{n} Y$. Let $\left\{{ }^{n} X_{t}, t \geq 0\right\}$ represent the inventory level process. We model it as a continuous-time Markov chain with the state space $\left\{\left({ }^{n} Y\right.\right.$, off $),\left({ }^{n} Y-1\right.$, off $), \ldots,(0$, off $),(0$, on $), \ldots,\left({ }^{n} Y-1\right.$, on $\left.)\right\}$, where $(i$, off $)$ indicates that the inventory level is $i$ and the production is off and the denotation of $(i$, on $)$ can be understood in the same way. Its transition rates are given by

$$
\begin{aligned}
& { }^{n} A_{(0, \text { off }),(0, \text { on })}=n \kappa, \quad{ }^{n} A_{(0, \text { off }),(0, \text { off })}=-n \mathcal{\kappa}, \\
& { }^{n} A_{(0, \text { on }),(1, \text { on })}=n \lambda(0), \quad{ }^{n} A_{(0, \text { on }),(0, \text { on })}=-n \lambda(0), \\
& { }^{n} A_{\left({ }^{n} Y-1, \text { on }\right),\left({ }^{n} Y, \text { off }\right)}=n \lambda\left(\frac{{ }^{n} Y-1}{n}\right), \quad{ }^{n} A_{\left({ }^{n} Y-1, \text { on }\right),\left({ }^{(n} Y-2, \text { on }\right)}=n \mu\left(\frac{{ }^{n} Y-1}{n}\right), \\
& { }^{n} A_{(n Y-1, \text { on }),\left({ }^{n} Y-1, \text { on }\right)}=-n \lambda\left(\frac{{ }^{n} Y-1}{n}\right)-n \mu\left(\frac{{ }^{n} Y-1}{n}\right),
\end{aligned}
$$

for all $i=1, \ldots,{ }^{n} Y$ :

$$
{ }^{n} A_{(i, \text { off }),(j, \text { off })}= \begin{cases}n \mu\left(\frac{i}{n}\right), & \text { if } j=i-1, \\ -n \mu\left(\frac{i}{n}\right), & \text { if } j=i,\end{cases}
$$

and finally for all $i=1, \ldots,{ }^{n} Y-2$ :

$$
{ }^{n} A_{(i, \text { on }),(j, \text { on })}= \begin{cases}n \mu\left(\frac{i}{n}\right), & \text { if } j=i-1 \\ n \lambda\left(\frac{i}{n}\right), & \text { if } j=i+1 \\ -n \mu\left(\frac{i}{n}\right)-n \lambda\left(\frac{i}{n}\right), & \text { if } j=i,\end{cases}
$$

where $n \lambda(i / n)$ and $n \mu(i / n)$ stand for the instantaneous production and demand rates, $n \kappa$ is the parameter of the exponentially distributed lead time between the switching and the 
actual production-on, and we have ignored all the cases when the transition rates take zero. So we have

$$
{ }^{n} \mathrm{TCU}\left({ }^{n} Y\right) \triangleq \lim _{T \rightarrow \infty} \frac{1}{T} E_{n} Y\left[\int_{0}^{T}\left\{g\left(\frac{{ }^{n} X_{t}}{n}\right)+K n \mu\left(\frac{1}{n}\right) I\left\{{ }^{n} X_{t}=1\right\}\right\} d t\right],
$$

with the holding cost $g(i / n)$ and setup cost $K>0$. Let us denote by ${ }^{n} Y^{*}$ the EIBL for the (scaled) stochastic model, so that ${ }^{n} \mathrm{TCU}\left({ }^{n} Y^{*}\right)=\inf _{n} Y=1,2, \ldots{ }^{n} \mathrm{TCU}\left({ }^{n} Y\right)$.

The concept of AFO and AO inventory backup level [ny] can be understood in the same manner as introduced at the end of Section 2.1.

\subsection{Main Results}

Condition 3. (a) There exist some constants $d_{1} \geq 0, k_{1}>0, \delta>0, \delta_{\mu \lambda}>0, \tilde{\eta}>1$ and measurable functions $\mu(x), \lambda(x)$, and $g(x)$ defined on $[0, \infty)$ such that $\delta \leq \mu(x) \leq \mu(x)+\delta_{\mu \lambda} \leq \lambda(x) \leq k_{1}$, $\lambda(x)+\mu(x) \leq k_{1},|g(x)| \leq d_{1}$, and $\inf _{x>0}(\lambda(x) / \mu(x))=\tilde{\eta}$.

(b) There exist finite intervals $\left(0, x_{1}\right),\left(x_{1}, x_{2}\right), \ldots$ with $\lim _{j \rightarrow \infty} x_{j}=\infty$ such that on each of them $\lambda(x), \mu(x)$, and $g(x)$ are Lipschitz continuous with Lipschitz constants $d_{\lambda}, d_{\mu}$, and $d_{\mathrm{g}}$, respectively.

Note that Condition 3 implies that for any fixed $y>0$, there exists an integer $L$ (possibly $y$-dependent) and $L+1$ intervals $\left(0, x_{1}\right),\left(x_{1}, x_{2}\right), \ldots,\left(x_{L}, 3[y+1]\right)$ such that on each interval $1 / \mu(x), g(x) / \mu(x)$, and $1 /(\lambda(x)-\mu(x))$ are Lipschitz continuous with Lipschitz constant $d_{\mu} / \delta^{2},\left(k_{1} d_{g}+d_{1} d_{\mu}\right) / \delta^{2}$, and $\left(d_{\lambda}+d_{\mu}\right) / \delta_{\mu \lambda^{\prime}}^{2}$, respectively, and at the same time on each of these intervals belonging to $(0, y)$, functions (with respect to $x) g(y-x) /(\lambda(y-x)-\mu(y-x)$ ) and $1 /(\lambda(y-x)-\mu(y-x))$ are Lipschitz continuous with Lipschitz constants $\left(k_{1} d_{g}+d_{1} d_{\mu}\right) / \delta^{2}$ and $\left(d_{\lambda}+d_{\mu}\right) / \delta_{\mu \lambda}^{2}$, respectively. Let us now denote the common Lipschitz constant by $d_{2} \triangleq \max \left\{d_{\mu} / \delta^{2},\left(k_{1} d_{g}+d_{1} d_{\mu}\right) / \delta^{2},\left(d_{\lambda}+d_{\mu}\right) / \delta_{\mu \lambda}^{2}\right\}$.

Proposition 3.1. Under Condition 3, for any fixed (fluid) inventory backup level y $>0$,

$$
\left|{ }^{n} \operatorname{TCU}([n y])-\operatorname{tcu}(y)\right| \leq \frac{2 \bar{\delta} \kappa k_{1}\left(1+d_{1}\right)}{\underline{\delta}}\left\{\frac{B_{1}+B_{2} n \tilde{\eta}^{-2 n[y+1]}+\max \{g(0) / 2 \kappa, 1 / 2 \kappa\}}{2[n y] \kappa+k_{1}}\right\}
$$

where one puts $\bar{\delta} \triangleq \max \left\{\delta, \delta_{\mu \kappa}\right\}$ and $\underline{\delta} \triangleq \min \left\{\delta, \delta_{\mu \kappa}\right\}$ and the $n$-independent terms $B_{1}, B_{2}$ are given by (A.2) in the appendix.

As in the case of EOQ models, we observe from Proposition 3.1 that [ $\left.n y^{*}\right]$ is AFO.

Corollary 3.2. Under Condition 3, the following statements hold. 
(a) If $g(x) \geq 0$, then $\underline{\lim }_{n \rightarrow \infty}\left({ }^{n} Y^{*} / n\right)>0$.

(b) If $\underline{\lim }_{n \rightarrow \infty}\left({ }^{n} Y^{*} / n\right)>0$, then $\left.\lim _{n \rightarrow \infty}\right|^{n} Y^{*} / n-\left[n y^{*}\right] / n \mid=0$ and $\left.\lim _{n \rightarrow \infty}\right|^{n} \mathrm{TCU}\left(\left[n y^{*}\right]\right)$

$-{ }^{n} \mathrm{TCU}\left({ }^{n} Y^{*}\right) \mid=0$, that is, $\left[n y^{*}\right]$ is $A O$.

(c) If $L$ is $y$-independent and $g(x) \geq 0$, then the following two substatements hold.

(c1) For $y \geq K \underline{\delta}^{2} / 4 k_{1} d_{1}$ and big enough $n$ so that $n>\max \left\{4 k_{1} d_{1} / K \underline{\delta}^{2}, k_{1} / 2 \kappa-1\right\}$,

$\left|{ }^{n} \operatorname{TCU}([n y])-\operatorname{tcu}(y)\right|$

$$
\begin{aligned}
& \leq \frac{2 \bar{\delta} \kappa k_{1}\left(1+d_{1}\right)}{\underline{\delta}}\left\{\frac{\widehat{B}_{1}+\widehat{B}_{2} n \tilde{\eta}^{-2 n\left(K \underline{\delta}^{2} / 4 k_{1} d_{1}+1\right)}+\max \{g(0) / 2 \kappa, 1 / 2 \kappa\}}{2\left(n\left(K \underline{\delta}^{2} / 4 k_{1} d_{1}\right)-1\right) \kappa+k_{1}}\right\} \\
& \triangleq F(n)=O\left(\frac{1}{n}\right) .
\end{aligned}
$$

Here $F(n)$ is $y$-independent, and

$$
\begin{gathered}
\widehat{B}_{1}=\frac{k_{1} d_{2}(\tilde{\eta}+1) 3\left(K \underline{\delta}^{2} / 4 k_{1} d_{1}+1\right)}{\delta(\tilde{\eta}-1)}+\left(1+\frac{k_{1} \tilde{\eta}}{\delta(\tilde{\eta}-1)}\right) \frac{3 d_{1} L(\tilde{\eta}+1)}{\delta(\tilde{\eta}-1)}, \\
\widehat{B}_{2}=\left(1+\frac{6 k_{1}\left(K \underline{\delta}^{2} / 4 k_{1} d_{1}+1\right) \tilde{\eta}}{\delta(\tilde{\eta}-1)}\right) \frac{d_{1} \tilde{\eta}^{2}(\tilde{\eta}+1)}{\delta(\tilde{\eta}-1)^{2}}
\end{gathered}
$$

One has (c2) $\left|{ }^{n} \mathrm{TCU}\left({ }^{n} Y^{*}\right)-{ }^{n} \mathrm{TCU}\left(\left[n y^{*}\right]\right)\right| \leq 2 F(n)=O(1 / n)$.

\section{Example and Comments}

In this section we firstly verify our results by considering a specific EPQ model, where tcu( $(\cdot)$ and ${ }^{n} \mathrm{TCU}(\cdot)$ can be analytically computed. Then we comment on the applications of our results.

Example 4.1. As for EOQ models, one may refer to [14] for an example. Hence we study the following classic setting for the (scaled) stochastic EPQ model: assume constant demand and production rates $\mu(x) \triangleq D>0$ and $\lambda(x) \triangleq R>0$, linear holding cost $g(x)=h x$ with a constant $h>0$, constant setup cost $K>0$, and finally no lead time between "switching" and "actual production-on," corresponding to if we take $\mathcal{\kappa} \rightarrow \infty$. Therefore, if we consider the underlying continuous time Markov chain $\left\{{ }^{n} X_{t}, t \geq 0\right\}$, state $(0$, off) will be excluded. The transition rates are modified accordingly. Clearly, Condition 3 is satisfied with this classic setting. The following lemma gives the explicit formula for ${ }^{n} \mathrm{TCU}(Z)$, where for simplicity we have put $Z$ instead of ${ }^{n} Y$ for the inventory backup level. 
Proposition 4.2. For the stochastic EPQ model described above,

${ }^{n} \mathrm{TCU}(Z)$

$$
\begin{aligned}
= & \left\{R h Z^{2}(D-R)^{2}+2 K n^{2} D(R-D)^{3}+Z h R(3 D-R)(D-R)+2 R h D^{2}\left(1-\left(\frac{D}{R}\right)^{Z}\right)\right\} \\
& \times \frac{1}{2 n(R-D)\left\{Z R^{2}-D^{2}+D^{2}(D / R)^{Z}-Z R D\right\}} .
\end{aligned}
$$

The proof of this proposition is based on solving (quite tediously) the associated Poisson equation for ${ }^{n} \mathrm{TCU}(Z)$ and is omitted here.

The corresponding deterministic EPQ model can be solved easily, and we have

$$
\operatorname{tcu}(y)=\frac{K D(R-D)}{y R}+\frac{h y}{2}
$$

Clearly, if we put $Z=[n y]$ in the expression for ${ }^{n} \mathrm{TCU}(Z)$, where $y$ is the inventory backup level for the fluid model, then one can easily see that

$$
\begin{gathered}
\lim _{n \rightarrow \infty}{ }^{n} \operatorname{TCU}([n y])=\frac{K D(R-D)}{y R}+\frac{h y}{2}=\operatorname{tcu}(y), \\
\left|\operatorname{tcu}(y)-{ }^{n} \operatorname{TCU}([n y])\right|=O\left(\frac{1}{n}\right),
\end{gathered}
$$

which agree with Proposition 3.1. For the means of illustration, we put $K=5, h=1, D=5$, $R=10$. Then the graphs of tcu( $(\cdot)$ and ${ }^{n} \mathrm{TCU}(\cdot)$ are plotted in Figure 1. Note that when $n=100$, the curves of tcu(y) and ${ }^{n} \mathrm{TCU}([n y])$ nearly coincide.

Secondly, if $n$ increases, then by inspecting the numerator (especially the first two terms) and the denominator of the expression for ${ }^{n} \mathrm{TCU}(Z)$, we see that if $Z$ does not increase as fast as $n,{ }^{n} \mathrm{TCU}(Z)$ will blow up to $\infty$ (it can be easily checked that the expression $\left.Z R^{2}-D^{2}+D^{2}(D / R)^{Z}-Z R D>0\right)$. Therefore, the condition of $\underline{\lim }_{n \rightarrow \infty}\left({ }^{n} Y^{*} / n\right)>0$ in Corollary 3.2 is satisfied.

\section{Comments}

Let us comment on the applicability issues of our main results (Propositions 2.2 and 3.1). We mainly focus on EOQ models, as absolutely similar comments can be made on EPQ models in the same manner.

Although we assume the ordering point to be always zero, our results are still applicable when it is set to be some fixed positive level, because Lemma A.1 holds if we put another absorbing state instead of zero. In particular, if one allows the state taking negative values, by putting some negative state absorbing, our results also impound the case of backlogging. 


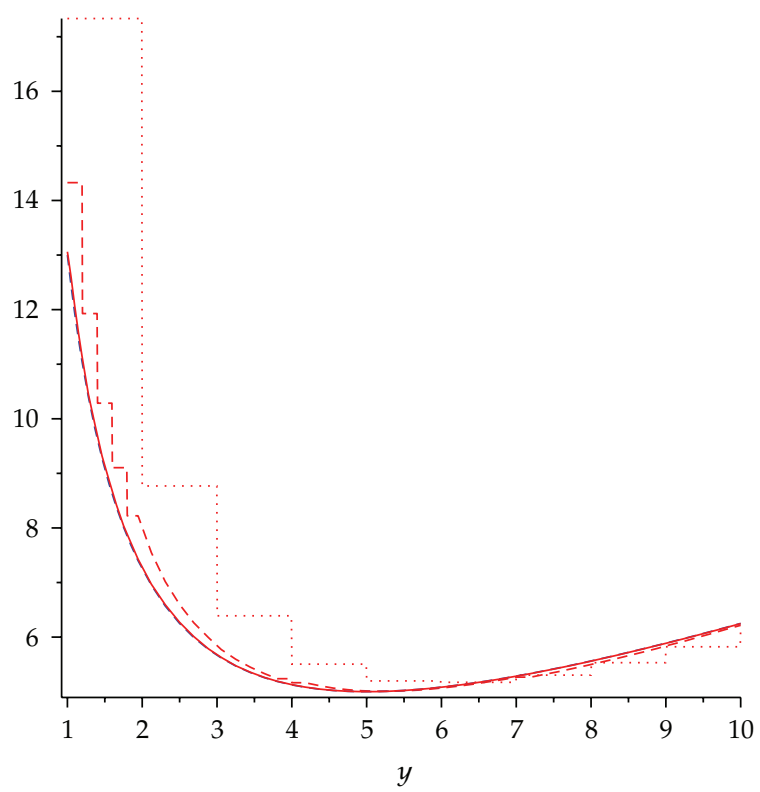

Figure 1: The dotted line (resp., dashed line, solid line) corresponds to ${ }^{1} \mathrm{TCU}([y])$ (resp., ${ }^{5} \mathrm{TCU}([5 y])$, $\operatorname{tcu}(y))$.

This flexibility regarding the ordering point together with the fact that $g(\cdot)$ is unrestricted in signs enriches the applications of our results, in that although we require $\mu$ to be separated from zero, when profit rather than solely operational cost is counted, the ordering point is most likely positive, meaning in cases of $\mu(x)=\alpha x^{\beta}, \alpha>0,0<\beta<1$ as in [20] and $\mu(x)=\alpha x^{-\beta}, \alpha>0, \beta \geq 1$ as in [1], $\mu$ will be essentially separated from zero, validating our results.

The state-dependence given in Conditions 1 and 3 is fairly general. In particular, that functions $\lambda(x), \mu(x)$ and $g(x)$ being bounded is not restrictive, because once some EOQ for the fluid model $y>0$ is fixed, to validate Propositions 2.2 and 3.1, they are only required to be bounded on bounded intervals. Note that in addition to the demand rate, some authors such as those of $[5,6]$ also include a state-dependent deteriorating rate, to indicate that the underlying goods are perishable. Our results are also applicable to such cases: one only needs explain $\mu(\cdot)$ as the total reduction rate of the inventory level.

Finally, Propositions 2.2 and 3.1 are significant extensions of the relevant results in [14], where the author only focuses on EOQ models and requires global Lipschitz continuity of $\mu(\cdot)$ and $g(\cdot)$. However, from the modelling point of view, the case of discontinuous functions is interesting and important as demonstrated by [1, 3, 5, 21, 22], where [1] considers a piecewise constant function $\mu(\cdot)$ and the others consider discontinuous $\mu(\cdot)$ taking either a constant value or according to $\mu(x)=\alpha x^{\beta}, \alpha>0,0<\beta<1$. The results in [14] are derived based on the closed form of the solution to a Poisson equation satisfied by ${ }^{n} \mathrm{TCU}(\cdot)$, which is tremendously difficult to get explicitly in the case of (stochastic) EPQ models. 


\section{Conclusions}

To sum up, in this work we formally justified a general class of inventory level-dependent deterministic EOQ and EPQ models, regarded as the fluid approximations to their stochastic versions, by showing a translation of the fluid EOQ (EIBL) to provide an order quantity (inventory backup level) asymptotically achieving some optimality for the stochastic model. The efficiency of the translation was obtained, as distinguished from the majority of the works on fluid approximations. The class of inventory models are quite broad so that to various extent, the obtained results are directly applicable to the existing works such as $[1,5,6,8,20-22]$. The present work is a significant extension of the relevant results in [14].

\section{Appendix}

To aid our proof, firstly, let us consider the following one-dimensional birth-and-death process $\left\{{ }^{n} Z_{t}, t \geq 0\right\}$ with state space $\{0,1, \ldots\}$ and birth and death rates $n \alpha(i / n)$ and $n \beta(i / n)$, respectively, where nonnegative measurable functions $\alpha$ and $\beta$ are defined on $[0, \infty)$ and $i$ indicates the current state of the process. In addition, $\alpha(0)=\beta(0)=0$, where the equality holds only at 0 , meaning that state zero is absorbing. Let $E_{i}$ denote the expectation of any underlying functional of the process with the initial state ${ }^{n} Z_{0}=i$. Let a real measurable function $\gamma(\cdot)$ defined on $[0, \infty)$ be fixed with $\gamma(0)=0$. Now we are in the position to state the following condition.

Condition 1. (a) There exist constants $\tilde{\eta}>1, \delta>0, d_{1}>0$, and $k_{1}<\infty$ such that $\inf _{z>0}(\beta(z) / \alpha(z))>\tilde{\eta}, \beta(z) \geq \delta, \alpha(z)+\beta(z) \leq k_{1},|\gamma(z)| \leq d_{1}$. Here if $\alpha(z) \equiv 0$, then $\tilde{\eta}$ can be arbitrary.

(b) There exist finite intervals $\left(z_{0} \triangleq 0, z_{1}\right),\left(z_{1}, z_{2}\right), \ldots$ with $\lim _{j \rightarrow \infty} z_{j}=\infty$ such that on each of them, $\gamma(z) /(\beta(z)-\alpha(z))$ is a Lipschitz continuous function with a common Lipschitz constant $d_{2}$.

Note that Condition 1(b) implies that for any fixed $y>0$ there exists an integer $L$ (possibly $\widehat{z}$-dependent) and $L+1$ finite intervals $\left(0, z_{1}\right),\left(z_{1}, z_{2}\right), \ldots,\left(z_{L}, 3[y+1]\right)$ such that on each interval, function $\gamma(\cdot) /(\beta-\alpha)$ is Lipschitz continuous with a common Lipschitz constant $d_{2}$.

The following lemma is a slightly stronger version of [15, Theorem 2] and will play an important role in our proof.

Lemma A.1. Suppose that Condition 1 is satisfied. Then for each $y>0$

$$
\sup _{0 \leq i \leq n[y+1]}\left|E_{i}\left[\int_{0}^{\infty} r\left(\frac{{ }^{n} Z_{s}}{n}\right) d s\right]-\int_{0}^{\infty} r(z(s)) d s\right| \leq \frac{B_{1}}{n}+B_{2} \tilde{\eta}^{-2 n[y+1]},
$$

where regarding the second integral the underlying dynamics is given by $d z / d s=\alpha(z)-\beta(z)$, 
$z(0)=i / n$ and $B_{1}$ and $B_{2}$ are given by

$$
\begin{gathered}
B_{1}=\frac{k_{1} d_{2}(\tilde{\eta}+1) 3[y+1]}{\delta(\tilde{\eta}-1)}+\left(1+\frac{k_{1} \tilde{\eta}}{\delta(\tilde{\eta}-1)}\right) \frac{3 d_{1} L(\tilde{\eta}+1)}{\delta(\tilde{\eta}-1)}, \\
B_{2}=\left(1+\frac{6 k_{1}[y+1] \tilde{\eta}}{\delta(\tilde{\eta}-1)}\right) \frac{d_{1} \tilde{\eta}^{2}(\tilde{\eta}+1)}{\delta(\tilde{\eta}-1)^{2}} .
\end{gathered}
$$

Proof. It can be easily checked in the proof of [14, Theorem 2] that our Condition 1, weaker than the original conditions imposed therein, is sufficient for the statement. See also [23].

\section{Proofs of Proposition 2.2, Corollaries 2.4 and 2.5, and Proposition 2.6}

For both the fluid model and (scaled) stochastic model let us call the time duration between two consecutive replenishments a cycle and denote them by $t_{\text {cycle }}$ and ${ }^{n} T_{\text {cycle, }}$, respectively. Here for simplicity, we do not explicitly indicate the $y$-dependence (resp., ${ }^{n} Y$-dependence) of $t_{\text {cycle }}\left(\right.$ resp., ${ }^{n} T_{\text {cycle }}$ ). Clearly $\left\{{ }^{n} X_{t}, t \geq 0\right\}$ is a regenerative process [24, page 425] in that it probabilistically repeats itself from one cycle to the next. It then follows from [25, Theorem 1.1, Proposition 131] (see also [24, Proposition 7.3]) that as far as the long-run average ${ }^{n} \mathrm{TCU}([n y])$ is concerned, it suffices to consider the inventory level process and the cost incurred with it over only one cycle. For simplicity, we always consider the cycle starting at time $t=0$ with the initial position ${ }^{n} X_{0}=[n y]$. Let us denote by ${ }^{n} \mathrm{TC}$ and tc the total cost incurred over the cycle in the stochastic and fluid model, respectively. Then the following lemma indicates that the difference between $E_{[n y]}\left[{ }^{n} T_{\text {cycle }}\right]$ and $t_{\text {cycle }}$ and the one between $E_{[n y]}\left[{ }^{n} \mathrm{TC}\right]$ and tc cannot be too big.

Lemma A.2. Under Condition 1 the following two inequalities hold with nonnegative $B_{1}$ and $B_{2}$ given by (A.2):

(a) $\left|E_{[n y]}\left[{ }^{n} T_{\text {cycle }}\right]-t_{\text {cycle }}\right| \leq B_{1} / n+B_{2} \tilde{\eta}^{-n[y+1]}+1 / n \kappa$;

(b) $\left|E_{[n y]}\left[{ }^{n} \mathrm{TC}\right]-\mathrm{tc}\right| \leq B_{1} / n+B_{2} \tilde{\eta}^{-n[y+1]}+g(0) / n \kappa$.

Proof. (a) Let us denote by ${ }^{n} T_{\text {absorbing }}$ the time duration from the starting point $t=0$ up to the point when ${ }^{n} X_{t}$ firstly reaches state zero. Obviously we have

$$
E_{[n y]}\left[{ }^{n} T_{\text {absorbing }}\right]=E_{[n y]}\left[\int_{0}^{\infty} I\left\{{ }^{n} X_{t}>0\right\} d t\right] .
$$

Then $E_{[n y]}\left[{ }^{n} T_{\text {cycle }}\right]=E_{[n y]}\left[{ }^{n} T_{\text {absorbing }}\right]+1 / n \kappa$, where the second term on the right hand side is the expected lead time. Now observe firstly that Condition 1 is a specific version of Condition 1: one can take $\tilde{\eta}>1$ to be arbitrary, and put functions (Here it does no matter to put $\mu(0)=0$.) $\alpha(x) \equiv 0, \beta(x)=\mu(x)$ and $I\{x>0\}=g(x)=\gamma(x)$; and secondly that the inventory level process from $t=0$ up to ${ }^{n} T_{\text {absorbing }}$ is a pure death process. Therefore, one can refer to Lemma A.1 for $\left|E_{[n y]}\left[{ }^{n} T_{\text {cycle }}\right]-t_{\text {cycle }}\right| \leq\left|E_{[n y]}\left[{ }^{n} T_{\text {absorbing }}\right]-t_{\text {cycle }}\right|+1 / n \kappa \leq$ $B_{1} / n+B_{2} \tilde{\eta}^{-n[y+1]}+1 / n \kappa$.

(b) Let us denote by ${ }^{n} \mathrm{TC}_{\text {absorbing }}$ the cost incurred during the interval $\left[0,{ }^{n} T_{\text {absorbing }}\right]$, so that $E_{[n y]}\left[{ }^{n} \mathrm{TC}\right]=E_{[n y]}\left[{ }^{n} \mathrm{TC}_{\text {absorbing }}\right]+g(0) / n \mathcal{K}$, where the second term on the right hand side 
corresponds to the cost incurred over the lead time. In the same way as in part (a), comparing $E_{[n y]}\left[{ }^{n} \mathrm{TC}_{\mathrm{absorbing}}\right]$ with tc first, and then adding $g(0) / n \kappa$ results in the statement. Remember that the setup cost cancels out.

Proof of Proposition 2.2. Under Condition 1 we have

$$
\begin{aligned}
\left|{ }^{n} \mathrm{TCU}([n y])-\mathrm{tcu}(y)\right| & =\left|\frac{E_{[n y]}\left[{ }^{n} \mathrm{TC}\right]}{E_{[n y]}\left[{ }^{n} T_{\text {cycle }}\right]}-\frac{\mathrm{tc}}{t_{\text {cycle }}}\right|=\left|\frac{E_{[n y]}\left[{ }^{n} \mathrm{TC}\right] t_{\text {cycle }}-\mathrm{tc} E_{[n y]}\left[{ }^{n} T_{\text {cycle }}\right]}{E_{[n y]}\left[{ }^{n} T_{\text {cycle }}\right] t_{\text {cycle }}}\right| \\
& =\left|\frac{E_{[n y]}\left[{ }^{n} \mathrm{TC}\right] t_{\text {cycle }}-\mathrm{tc} t_{\text {cycle }}+\mathrm{tc} t_{\text {cycle }}-\mathrm{tc} E_{[n y]}\left[{ }^{n} T_{\text {cycle }}\right]}{E_{[n y]}\left[{ }^{n} T_{\text {cycle }}\right] t_{\text {cycle }}}\right| \\
& =\left|\frac{t_{\text {cycle }}\left\{E_{[n y]}\left[{ }^{n} \mathrm{TC}\right]-\mathrm{tc}\right\}+\mathrm{tc}\left\{t_{\text {cycle }}-E_{[n y]}\left[{ }^{n} T_{\text {cycle }}\right]\right\}}{E_{[n y]}\left[{ }^{n} T_{\text {cycle }}\right] t_{\text {cycle }}}\right| \\
& \leq \frac{t_{\text {cycle }}\left|E_{[n y]}\left[{ }^{n} \mathrm{TC}\right]-\mathrm{tc}\right|+\mathrm{tc} \mid t_{\text {cycle }}-E_{[n y]}\left[{ }^{n} T_{\text {cycle }}\right]}{E_{[n y]}\left[{ }^{n} T_{\text {cycle }}\right] t_{\text {cycle }}} \\
& \leq \frac{\left(y / \delta+d_{1} y / \delta\right)}{\left(y / k_{1}\right)\left([n y] / n k_{1}+1 / n \kappa\right)}\left\{\frac{B_{1}}{n}+B_{2} \tilde{\eta}^{-2 n[y+1]}+\max \left\{\frac{1}{n \kappa}, \frac{g(0)}{n \kappa}\right\}\right\} \\
& =\frac{\left(1+d_{1}\right) n k_{1}^{2} \kappa}{\delta\left([n y] \kappa+k_{1}\right)}\left\{\frac{B_{1}}{n}+B_{2} \tilde{\eta}^{-2 n[y+1]}+\max \left\{\frac{1}{n \kappa}, \frac{g(0)}{n \kappa}\right\}\right\},
\end{aligned}
$$

where the last inequality follows from the facts that $y / k_{1} \leq t_{\text {cycle }} \leq y / \delta$, tc $\leq d_{1} y / \delta$, $E_{[n y]\left[{ }^{n} T_{\text {cycle }}\right]} \geq[n y] / n k_{1}+1 / n \kappa$ and Lemma A.2.

Now let us easily observe that $(\tilde{\eta}+1) /(\tilde{\eta}-1)$ and $\tilde{\eta} /(\tilde{\eta}-1)$ both decrease with $\tilde{\eta} \in(1, \infty)$. It follows that $B_{1} / n, B_{2} \tilde{\eta}^{-2 n[y+1]}$, and thus the above-derived expression all decrease with $\tilde{\eta}$, where we recall that $\tilde{\eta}$ can be an arbitrary number on the interval $(1, \infty)$, see Condition 1 . This implies that

$$
\begin{aligned}
\mid{ }^{n} & \operatorname{TCU}([n y])-\operatorname{tcu}(y) \mid \\
& \leq \lim _{\tilde{\eta} \rightarrow \infty} \frac{\left(1+d_{1}\right) k_{1}^{2} n \mathcal{\kappa}}{\delta\left([n y] \kappa+k_{1}\right)}\left\{\frac{B_{1}}{n}+B_{2} \tilde{\eta}^{-2 n[y+1]}+\max \left\{\frac{1}{n \kappa}, \frac{g(0)}{n \kappa}\right\}\right\} \\
& =\frac{\left(1+d_{1}\right) k_{1}^{2} \kappa}{\delta\left([n y] \kappa+k_{1}\right)}\left\{\frac{k_{1} d_{2} 3[y+1]}{\delta}+\left(1+\frac{k_{1}}{\delta}\right) \frac{3 d_{1} L}{\delta}+\max \left\{\frac{g(0)}{\kappa}, \frac{1}{\kappa}\right\}\right\} .
\end{aligned}
$$

Proof of Corollary 2.4. For any fixed $n$, let us denote ${ }^{n} Y^{*}=n \widehat{y}(n)$. We do the proof in two parts.

Part 1. We consider the case of a convergent sequence $\hat{y}(n)$. Suppose now that as $n \rightarrow \infty$, $\widehat{y}(n)$ does not go to $y^{*}$ but $\lim _{n \rightarrow \infty} \widehat{y}(n)=\tilde{y}>0$; here we allow $\tilde{y}$ to be from the extended real line. In particular, for big enough $n, \widehat{y}(n)$ is separated from zero. According to Proposition 2.2, we have ${ }^{n} \operatorname{TCU}(n \hat{y}(n)) \rightarrow \operatorname{tcu}(\widehat{y}(n))$. But we also have $\operatorname{tcu}(\widehat{y}(n)) \rightarrow \operatorname{tcu}(\tilde{y})$, since 
$\operatorname{tcu}(y)=\left(\int_{0}^{y}(g(x) / \mu(x) d x)+K\right) / \int_{0}^{y}(d x / \mu(x))$ is continuous in $y$. This gives ${ }^{n} \operatorname{TCU}(n \widehat{y}(n)) \rightarrow$ $\operatorname{tcu}(\tilde{y})>\operatorname{tcu}\left(y^{*}\right)$. However, it follows from Proposition 2.2 that ${ }^{n} \mathrm{TCU}\left(\left[n y^{*}\right]\right) \rightarrow \operatorname{tcu}\left(y^{*}\right)$. This indicates that at least for big enough $n,{ }^{n} \mathrm{TCU}\left(\left[n y^{*}\right]\right)<{ }^{n} \mathrm{TCU}(n \widehat{y}(n))={ }^{n} \mathrm{TCU}\left({ }^{n} Y^{*}\right)$, which is a desired contradiction. Hence $\lim _{n \rightarrow \infty} \widehat{y}(n)=y^{*}$, and consequently, $\left.\lim _{n \rightarrow \infty}\right|^{n} \mathrm{TCU}\left(\left[n y^{*}\right]\right)-$ ${ }^{n} \mathrm{TCU}\left({ }^{n} Y^{*}\right) \mid=0$, as required.

Part 2. Now consider the case of a divergent sequence $\widehat{y}(n)$. One only needs consider the following two situations: either it has a bounded subsequence, which by BolzanoWeierstrass theorem further has a convergent subsequence; or it does not have a bounded subsequence, which means that it has a subsequence blowing up to $\infty$. However, by taking the corresponding subsequences, we find that both situations have been essentially covered in Part 1. Part 2 is thus proved.

Proof of Corollary 2.5. (a) Under the conditions of the statement we have

RHS of (2.7)

$$
\begin{aligned}
& \leq \frac{\left(1+d_{1}\right) k_{1}^{2}}{\delta[n y]}\left\{\frac{k_{1} d_{2}(3 y+3)}{\delta}+\left(1+\frac{k_{1}}{\delta}\right) \frac{3 d_{1} L}{\delta}+\max \left\{\frac{g(0)}{\kappa}, \frac{1}{\mathcal{K}}\right\}\right\} \\
& =\frac{\left(1+d_{1}\right) k_{1}^{3} 3 d_{2} y}{\delta^{2}[n y]}+\frac{\left(1+d_{1}\right) k_{1}^{2}}{\delta[n y]}\left\{\frac{3 k_{1} d_{2}}{\delta}+\left(1+\frac{k_{1}}{\delta}\right) \frac{3 d_{1} L}{\delta}+\max \left\{\frac{g(0)}{\mathcal{K}}, \frac{1}{\mathcal{K}}\right\}\right\} \\
& \leq \frac{3\left(1+d_{1}\right) k_{1}^{3} d_{2}}{\delta^{2}} \frac{y}{n y-1}+\frac{\left(1+d_{1}\right) k_{1}^{2}}{\delta(n y-1)}\left\{\frac{3 k_{1} d_{2}}{\delta}+\left(1+\frac{k_{1}}{\delta}\right) \frac{3 d_{1} L}{\delta}+\max \left\{\frac{g(0)}{\kappa}, \frac{1}{\mathcal{K}}\right\}\right\}
\end{aligned}
$$

(Recall, here $n y-1>0)$

$$
\begin{aligned}
\leq & \frac{3\left(1+d_{1}\right) k_{1}^{3} d_{2}}{\delta^{2}} \frac{1}{n\left\{1-\kappa k_{1} d_{1} /\left(n \kappa \delta^{2} K-\delta k_{1} d_{1}\right)\right\}}+\frac{\left(1+d_{1}\right) k_{1}^{2}}{n \delta\left\{\delta^{2} K / k_{1} d_{1}-\delta / n \kappa-1 / n\right\}} \\
& \times\left\{\frac{3 k_{1} d_{2}}{\delta}+\left(1+\frac{k_{1}}{\delta}\right) \frac{3 d_{1} L}{\delta}+\max \left\{\frac{g(0)}{\kappa}, \frac{1}{\mathcal{K}}\right\}\right\} .
\end{aligned}
$$

(Here we use the fact that $y /(n y-1)$ decreases with $y$ and $y \geq \delta^{2} K / k_{1} d_{1}-\delta / n \kappa$.) (a) is now clear.

(b) According to Lemma 2.3, $y^{*} \geq \delta^{2} K / k_{1} d_{1}$, and ${ }^{n} Y^{*}$ satisfies ${ }^{n} Y^{*} / n \geq \delta^{2} K / k_{1} d_{1}-$ $\delta / n \kappa$. Therefore, according to part (a), for $n \geq N$ we have

$$
{ }^{n} \mathrm{TCU}\left(\left[n y^{*}\right]\right) \leq \operatorname{tcu}\left(y^{*}\right)+E(n) \leq \operatorname{tcu}\left(\frac{{ }^{n} Y^{*}}{n}\right)+E(n) \leq{ }^{n} \mathrm{TCU}\left({ }^{n} Y^{*}\right)+2 E(n)
$$


in one direction and

$$
{ }^{n} \operatorname{TCU}\left(\left[n y^{*}\right]\right) \geq{ }^{n} \operatorname{TCU}\left({ }^{n} Y^{*}\right) \geq \operatorname{tcu}\left(\frac{{ }^{n} Y^{*}}{n}\right)-E(n) \geq \operatorname{tcu}\left(y^{*}\right)-2 E(n)
$$

in the other direction. Combining both directions results in the statement.

Proof of Proposition 2.6. Now ${ }^{n} \Pi(i)$ and $\pi(x)$ can be easily computed as done in [14]. So we have

$$
\begin{gathered}
\Pi(0)=\frac{1 / n \mathcal{K}}{\sum_{j=1}^{[n y]}(1 / n \mu(j / n)+1 / n \kappa)}, \quad \Pi(i)=\frac{1 / n \mu(i / n)}{\sum_{j=1}^{[n y]}(1 / n \mu(j / n)+1 / n \kappa)}, \\
i=1,2, \ldots,[n y] ; \\
\pi(0)=0 ; \quad \pi(x)=\frac{1}{\mu(x) t_{\mathrm{cycle}}}, \quad 0<x \leq[n y] .
\end{gathered}
$$

Here we put $\pi(0)=0$ for convenience. Then $\left|{ }^{n} \Pi(0)-\pi(0)\right| \leq 1 / n \kappa\left(1 / n \kappa+[n y] / n k_{1}\right) \leq$ $k_{1} /\left(k_{1}+\kappa[n y]\right)$, and

$$
\begin{aligned}
\mid n & \Pi(i)-\int_{(i-1) / n}^{i / n} \pi(x) d x \mid \\
= & \left|\frac{1 / n \mu(i / n)}{E_{[n y]}\left[{ }^{n} T_{\text {cycle }}\right]}-\frac{\int_{(i-1) / n}^{i / n}(1 / \mu(x)) d x}{t_{\text {cycle }}}\right| \\
= & \mid \frac{1}{n \mu(i / n)} t_{\text {cycle }}-\int_{(i-1) / n}^{i / n} \frac{1}{\mu(x)} d x t_{\text {cycle }}+\int_{(i-1) / n}^{i / n} \frac{1}{\mu(x)} d x t_{\text {cycle }} \\
& -\int_{(i-1) / n}^{i / n} \frac{1}{\mu(x)} d x E_{[n y]}\left[T_{\text {cycle }]} \mid \frac{1}{E_{[n y][}{ }^{n} T_{\text {cycle }] t_{\text {cycle }}}}\right. \\
\leq & \frac{t_{\text {cycle }}\left|1 / n \mu(i / n)-\int_{(i-1) / n}^{i / n}(d x / \mu(x))\right|+\int_{(i-1) / n}^{i / n}(d x / \mu(x))\left|E_{[n y]}\left[{ }^{n} T_{\text {cycle }}\right]-t_{\text {cycle }}\right|}{E_{[n y]\left[{ }^{n} T_{\text {cycle }} t_{\text {cycle }}\right]}} \\
\leq & \frac{2 y / n \delta^{2}+(1 / n \delta)\left(B_{1} / n+B_{2} \tilde{\eta}^{-n 2[y+1]}+1 / n \kappa\right)}{\left([n y] / n k_{1}+1 / n \kappa\right) y / k_{1}} .
\end{aligned}
$$


(Here we recall (a) of Lemma A.2.) Recall that in the above derived expression, $\tilde{\eta}$ can be any number from $(1, \infty)$. After passing to the limit $\tilde{\eta} \rightarrow \infty$, we eventually end up with

$$
\begin{aligned}
& \left|{ }^{n} \Pi(i)-\int_{(i-1) / n}^{i / n} \pi(x) d x\right| \\
& \quad \leq \frac{2 y k_{1}^{2} \kappa / \delta^{2}+\left(k_{1}^{2} \kappa / n \delta\right)\left(3 k_{1} d_{2}(y+1) / \delta+\left(1+k_{1} / \delta\right)\left(3 d_{1} L / \delta\right)+1 / \kappa\right)}{([n y]+1) y},
\end{aligned}
$$

as required.

\section{Proofs of Proposition 3.1 and Corollary 3.2}

Let us call a cycle the time duration between two consecutive moments when the inventory is fully backed up. Arguing similarly as for EOQ models, it suffices to consider the inventory level process $\left\{{ }^{n} X_{t}, t \geq 0\right\}$ and the cost incurred over one complete cycle, for which we put the starting time of $t=0$. Let us denote by $t_{\text {cycle, }}{ }^{n} T_{\text {cycle }}$ and tc, ${ }^{n} \mathrm{TC}$ the duration of a cycle and the cost incurred over a cycle in the fluid and scaled stochastic model, respectively. Notice additionally that a cycle is always constituted to by two phases corresponding to the on and off of the production. This raises another set of denotations: let $t_{\text {on }},{ }^{n} T_{\text {on }}\left(t_{\text {off }},{ }^{n} T_{\text {off }}\right)$, and $\mathrm{tc}_{\mathrm{on}},{ }^{n} \mathrm{TC}_{\mathrm{on}}\left(\mathrm{tc}_{\mathrm{off}},{ }^{n} \mathrm{TC}_{\mathrm{off}}\right)$ be the total cost incurred during the production-on (off) phases in the fluid and (scaled) stochastic model, respectively. We agree on that in both fluid and (scaled) stochastic model the setup cost is accounted for in $\mathrm{tc}_{\text {off }}$ and ${ }^{n} \mathrm{TC}_{\text {off. }}$ Then obviously we have $t_{\text {cycle }}=t_{\text {on }}+t_{\text {off }}, E\left[{ }^{n} T_{\text {cycle }}\right]=E\left[{ }^{n} T_{\text {on }}\right]+E\left[{ }^{n} T_{\text {off }}\right]$ and $\mathrm{tc}=\mathrm{tc}_{\text {on }}+\mathrm{tc}_{\text {off }}$, $E\left[{ }^{n} \mathrm{TC}\right]=E\left[{ }^{n} \mathrm{TC}_{\mathrm{on}}\right]+E\left[{ }^{n} \mathrm{TC}_{\text {off }}\right]$. Here and below, for convenience we omit the subscript of the expectation operator.

Lemma A.3. Under Condition 3 the following inequalities hold:

(a) $\left|E\left[{ }^{n} T C_{\text {on }}\right]-t c_{\text {on }}\right| \leq B_{1} / n+B_{2} \tilde{\eta}^{-2 n[y+1]},\left|E\left[{ }^{n} T_{\text {on }}\right]-t_{\text {on }}\right| \leq B_{1} / n+B_{2} \tilde{\eta}^{-2 n[y+1]}$;

(b) with nonnegative $B_{1}$ and $B_{2}$ given by (A.2),

$$
\begin{gathered}
\left|E\left[{ }^{n} T C_{\text {off }}\right]-t c_{\text {off }}\right| \leq \frac{B_{1}}{n}+B_{2} \tilde{\eta}^{-2 n[y+1]}+\frac{g(0)}{n \kappa}, \\
\left|E\left[{ }^{n} T_{\text {off }}\right]-t_{\text {off }}\right| \leq \frac{B_{1}}{n}+B_{2} \tilde{\eta}^{-2 n[y+1]}+\frac{1}{n \kappa} .
\end{gathered}
$$

Proof. (a) Let us concentrate on the inventory level process over the production-on phase. In the fluid model, it appears convenient to reflect the trajectory $\left\{x(t), t \in\left(t_{\text {on }}, t_{\text {cycle }}\right)\right\}$ (corresponding to the solid curve in Figure 2) about the horizontal $t$-axis first, and then shift the resulting trajectory (corresponding to the curve of crosses in Figure 2) upwards by $y$ units, and finally further shift the resulting trajectory to the left by shifting the time by $t_{\text {off }}$ units to the right to get $\left\{\right.$ on $\left.\widehat{x}(t), t \in\left[0, t_{\mathrm{on}}\right]\right\}$ (corresponding to the curve of solid boxes in Figure 2). Note now, for $\left\{\right.$ on $\left.\widehat{x}(t), t \in\left[0, t_{\text {on }}\right]\right\}$ with on $\widehat{x}(0)=y$ the roles of production and demand have switched over: each produced unit reduces on $\widehat{x}$ by one unit, and each demanded unit increases on $\widehat{x}$ by one unit. More precisely, let us define the following functions: 


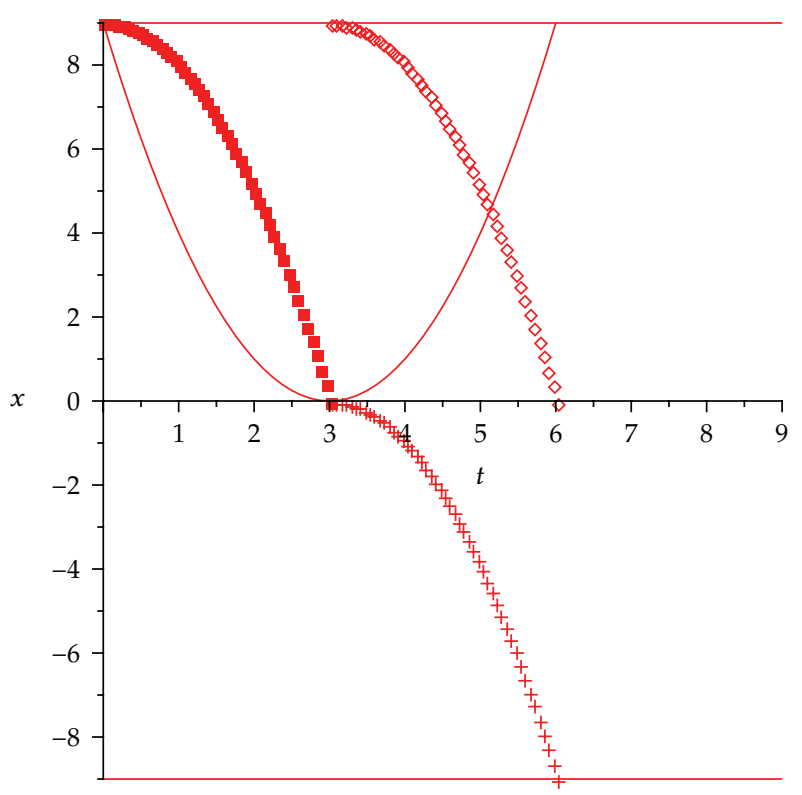

Figure 2: The illustrative graph of on $\widehat{x}(t)$.

$$
\begin{array}{ll}
\text { on } \widehat{\mu}(y)=\lambda(0), \quad \text { on } \widehat{\mu}(0)=0, \quad \text { on } \widehat{\mu}(x)=\lambda(y-x), & x \in(0, y), \\
\text { on } \widehat{\lambda}(y)=\mu(0)=0, \quad \text { on } \widehat{\lambda}(0)=0, \quad \text { on } \widehat{\jmath}=\mu(y-x), & x \in(0, y), \\
\text { on } \widehat{g}(y)=g(0), \quad \text { on } \widehat{g}(0)=0, \quad \text { on } \widehat{g}(x)=g(y-x), & x \in(0, y) .
\end{array}
$$

Then the dynamics of on $\widehat{x}(0)=y$ with $d_{\mathrm{on}} \widehat{x}(t) /\left.d t\right|_{\text {on } \widehat{x}(t)=x}={ }_{\text {on }} \hat{\lambda}(x)-{ }_{\text {on }} \widehat{\mu}(x)$ for $x \in(0, y]$ is of our interest, because we can write tc on $=\int_{0}^{\infty} g($ on $\widehat{x}(t)) d t$.

Absolutely similar arguments are applicable to the (scaled) stochastic model. Consequently, we can consider the inventory level process during a production-on phase as a birth-and-death process $\left\{\begin{array}{l}n \\ \text { on }\end{array} \widehat{X}_{t}, t \geq 0\right\}$ with initial condition ${ }_{\text {on }}^{n} \widehat{X}_{0}=[n y]$, state space $\{0,1, \ldots\}$, birth and death rates given by $n \alpha(i / n) \triangleq n_{\text {on }} \widehat{\lambda}(i / n)$ and $n \beta(i / n) \triangleq n_{\text {on }} \widehat{\mu}(i / n)$ when the current state is $i$, and the cost rate given by $\gamma(i / n) \triangleq{ }_{\text {on }} \hat{g}(i / n)$. By recognizing $E_{[n y]}\left[\int_{0}^{\infty}\right.$ on $\left.\widehat{g}\left({ }^{n}{ }_{\text {on }} \widehat{X}_{t}\right) d t\right]=E\left[{ }^{n} \mathrm{TC}_{\mathrm{on}}\right]$ and that Condition 3 is a specific version of Condition 1 , we can refer to Lemma A.1 for $\left|{ }^{n} E\left[{ }^{n} \mathrm{TC}_{\mathrm{on}}\right]-\mathrm{tc}_{\mathrm{on}}\right| \leq B_{1} / n+B_{2} \tilde{\eta}^{-2 n[y+1]}$. Arguing similarly as above (see also the proof of Lemma A.2), we have $\left|{ }^{n} E\left[{ }^{n} T_{\text {on }}\right]-t_{\text {on }}\right| \leq B_{1} / n+B_{2} \tilde{\eta}^{-2 n[y+1]}$. (a) is now clear.

(b) The production-off phase has already been covered when analyzing EOQ models. Therefore, one can directly refer to Lemma A.2 for the statement. 
Proof of Proposition 3.1. Lemma A.3 implies that

$$
\begin{aligned}
\left|E\left[{ }^{n} \mathrm{TC}\right]-\mathrm{tc}\right| & \leq\left|E\left[{ }^{n} \mathrm{TC}_{\mathrm{on}}\right]-\mathrm{tc}_{\mathrm{on}}\right|+\mid E\left[{ }^{n} \mathrm{TC} \text { off }\right]-\mathrm{tc}_{\mathrm{off}} \mid \\
& \leq \frac{2}{n}\left(B_{1}+B_{2} n \tilde{\eta}^{-2 n[y+1]}+\frac{g(0)}{2 \kappa}\right)
\end{aligned}
$$

and similarly $\left|E\left[{ }^{n} T_{\text {cycle }}\right]-t_{\text {cycle }}\right| \leq(2 / n)\left(B_{1}+B_{2} n \tilde{\eta}^{-2 n[y+1]}+1 / 2 \kappa\right)$. Then according to (A.4) and the facts of $E\left[{ }^{n} T_{\text {cycle }}\right] \geq 2[n y] / n k_{1}+1 / n \kappa, 2 y / \underline{\delta} \geq t_{\text {cycle }} \geq 2 y / \bar{\delta}$ and tc $\leq 2 y d_{1} / \underline{\delta}$ (recall $\bar{\delta} \triangleq \max \left\{\delta, \delta_{\mu \lambda}\right\}$ and $\left.\underline{\delta} \triangleq \min \left\{\delta, \delta_{\mu \lambda}\right\}\right)$, we have

$$
\begin{aligned}
\left|{ }^{n} \operatorname{TCU}([n y])-\operatorname{tcu}(y)\right| & \leq \frac{(2 y / \underline{\delta})(2 / n)\left\{B_{1}+B_{2} n \tilde{\eta}^{-2 n[y+1]}+\max \{g(0) / 2 \kappa, 1 / 2 \kappa\}\right\}\left(1+d_{1}\right)}{\left(2[n y] / n k_{1}+1 / n \kappa\right)(2 y / \bar{\delta})} \\
& =\frac{2 \bar{\delta} \kappa k_{1}\left(1+d_{1}\right)}{\underline{\delta}}\left\{\frac{B_{1}+B_{2} n \tilde{\eta}^{-2 n[y+1]}+\max \{g(0) / 2 \kappa, 1 / 2 \kappa\}}{2[n y] \kappa+k_{1}}\right\} .
\end{aligned}
$$

Proof of Corollary 3.2. (a) Suppose that the statement does not hold. That is, for some subsequence $\left\{n_{j}, j=1,2, \ldots\right\}$ with $n_{j} \rightarrow \infty$ as $j \rightarrow \infty,{ }^{n_{j}} Y^{*}=o\left(n_{j}\right)$ in that $\lim _{j \rightarrow \infty}\left({ }^{n_{j}} Y^{*} / n_{j}\right)=0$. Under ${ }^{n_{j}} Y^{*}$ we have

$$
\begin{aligned}
& E\left[{ }^{n_{j}} T_{\text {cycle }}\right] \\
& =\left\{\frac{1}{n_{j} \kappa}+\sum_{i=1}^{n_{j} Y^{*}} \frac{1}{n_{j} \mu\left(i / n_{j}\right)}\right\}+\sum_{i=0}^{n_{j} Y^{*}-1}\left\{\frac{1}{n_{j} \lambda\left(i / n_{j}\right)}+\sum_{k=0}^{i-1} \frac{\mu\left(i / n_{j}\right) \mu\left((i-1) / n_{j}\right) \cdots \mu\left((i-k) / n_{j}\right)}{n_{j} \lambda\left(i / n_{j}\right) \lambda\left((i-1) / n_{j}\right) \cdots \lambda\left((i-k-1) / n_{j}\right)}\right\},
\end{aligned}
$$

where by [26, Theorem 1, page 175] the term inside the first curry bracket corresponds to $E\left[{ }^{n_{j}} T_{\text {off }}\right]$ and the second (last) sum corresponds to $E\left[{ }^{n_{j}} T_{\text {on }}\right]$. Here we agree on that when $i=0$, the term in the second curry bracket reduces to $1 / n_{j} \lambda(0)$. This gives

$n_{j} \operatorname{TCU}\left({ }^{n_{j}} Y^{*}\right)$

$$
\begin{aligned}
& =\frac{E\left[{ }^{n_{j}} \mathrm{TC}\right]}{\left\{1 / n_{j} \mathcal{\kappa}+\sum_{i=1}^{n_{j} Y^{*}}\left(1 / n_{j} \mu\left(i / n_{j}\right)\right)\right\}+\sum_{i=0}^{n_{j} Y^{*}-1}\left\{1 / n_{j} \lambda\left(i / n_{j}\right)+\mathscr{A}\right\}} \\
& \geq \frac{K}{\left\{1 / n_{j} \mathcal{K}+\sum_{i=1}^{n_{j} Y^{*}} 1 / n_{j} \mu\left(i / n_{j}\right)\right\}+\sum_{i=0}^{n_{j} Y^{*}-1}\left\{1 / n_{j} \lambda\left(i / n_{j}\right)+\mathcal{A}\right\}}
\end{aligned}
$$

where $\mathcal{A}=\sum_{k=0}^{i-1}\left(\mu\left(i / n_{j}\right) \mu\left((i-1) / n_{j}\right) \cdots \mu\left((i-k) / n_{j}\right) / n_{j} \lambda\left(i / n_{j}\right) \lambda\left((i-1) / n_{j}\right) \cdots \lambda((i-k-\right.$ $\left.1) / n_{j}\right)$ ), where the last inequality follows from the fact $g(x) \geq 0$. Clearly, the right hand side expression of the above inequality goes to infinity as $n_{j} \rightarrow \infty$, because $\lambda(x)$ and $\mu(x)$ are 
both bounded and separated from zero, and ${ }^{n_{j}} Y^{*}=o\left(n_{j}\right)$ by supposition. On the other hand, obviously there exists some $y_{*}>0$ with tcu $\left(y_{*}\right)<\infty$, which according to Proposition 3.1 leads to that at least for big enough $n_{j},\left[n_{j} y_{*}\right]$ outperforms ${ }^{n_{j}} Y^{*}$, which is a desired contradiction. Part (a) is thus proved.

(b) The proof of this part coincides with the one of Corollary 2.4, and is thus omitted.

(c1) Let us notice first of all that under the conditions of the statement, we have that the expression $\left(\widetilde{B}_{1}+\widetilde{B}_{2} n \widetilde{\eta}^{-2 n(y+1)}+\max \{g(0) / 2 \kappa, 1 / 2 \kappa\}\right) /\left(2(n y-1) \kappa+k_{1}\right)$ is positive and decreases with $y$. Here $\widetilde{B}_{1}$ and $\widetilde{B}_{2}$ come from replacing $[y+1]$ by $y+1$ in $B_{1}$ and $B_{2}$. Indeed, as for the positivity part, one only needs to see the denominator $2(n y-1) \mathcal{K}+k_{1}>0$ if $n$ is subject to the given condition. The decreasing (with respect to $y$ ) part follows from $d\left[(y+1) /\left(2 \kappa(n y-1)+k_{1}\right)\right] / d y=\left(k_{2}-2 \kappa-2 \kappa n\right) /\left[2 \kappa(n y-1)+k_{1}\right]^{2}<0$ whenever $n>k_{1} / 2 \kappa-1$. Remember that $\widetilde{B}_{1}$ and $\widetilde{B}_{2}$ are both $y$-dependent, and $L$ is $y$-independent.

Now let us prove (c1) of the corollary. Observe that under the conditions of the statement, Proposition 3.1 implies that

$$
\begin{aligned}
& \left|E\left[{ }^{n} \operatorname{TCU}([n y])\right]-\operatorname{tcu}(y)\right| \\
& \quad \leq \frac{2 \bar{\delta} \kappa k_{1}\left(1+d_{1}\right)}{\underline{\delta}}\left\{\frac{\widetilde{B}_{1}+\widetilde{B}_{2} n \tilde{\eta}^{-2 n(y+1)}+\max \{g(0) / 2 \kappa, 1 / 2 \kappa\}}{2(n y-1) \kappa+k_{1}}\right\} .
\end{aligned}
$$

For $y \geq K \underline{\delta}^{2} / 4 k_{1} d_{1}$, one can bound from the above the right hand side expression in (A.18) by substituting $y=K \underline{\delta}^{2} / 4 k_{1} d_{1}$ in it, which leads to (c1).

(c2) Let us notice that $y^{*} \geq K \underline{\delta}^{2} / 4 k_{1} d_{1}$, and for big enough $n,{ }^{n} Y^{*} / n \geq K \underline{\delta}^{2} / 4 k_{1} d_{1}$. Indeed, due to (b), to justify the second inequality, we only need verify $y^{*} \geq K \underline{\delta}^{2} / 4 k_{1} d_{1}$, which is done as follows. For the fluid model, clearly we have

$$
\operatorname{tcu}(y)=\frac{\int_{0}^{y}(g(x) / \mu(x)) d x+\int_{0}^{y}(g(x) /(\lambda(x)-\mu(x)))+K}{\int_{0}^{y}(d x / \mu(x))+\int_{0}^{y}(d x /(\lambda(x)-\mu(x)))},
$$

where the numerator corresponds to tc and the denominator corresponds to $t_{\mathrm{cycle}}$, and

$$
\begin{aligned}
\frac{d \text { tcu }}{d y}= & \frac{(g(y) / \mu(y)+g(y) /(\lambda(y)-\mu(y)))\left(\int_{0}^{y}(d x / \mu(x))+\int_{0}^{y}(d x /(\lambda(x)-\mu(x)))\right)}{\left(\int_{0}^{y}(d x / \mu(x))+\int_{0}^{y}(d x /(\lambda(x)-\mu(x)))\right)^{2}} \\
& -\frac{\left(\int_{0}^{y}(g(x) / \mu(x)) d x+\int_{0}^{y}(g(x) /(\lambda(x)-\mu(x)))+K\right)(1 / \mu(y)+1 /(\lambda(y)-\mu(y)))}{\left(\int_{0}^{y}(d x / \mu(x))+\int_{0}^{y}(d x /(\lambda(x)-\mu(x)))\right)^{2}},
\end{aligned}
$$

which is negative if $(g(y) / \mu(y)+g(y) /(\lambda(y)-\mu(y)))\left(\int_{0}^{y}(d x / \mu(x))+\int_{0}^{y}(d x /(\lambda(x)-\mu(x)))\right)<$ $K / \mu(t)$. But the latter inequality holds if $y<K \underline{\delta}^{2} / 4 k_{1} d_{1}$, because $g(y) / \mu(y)+g(y) /(\lambda(y)-$ $\mu(y)) \leq 2 d_{1} / \underline{\delta}$ and $\int_{0}^{y}(d x / \mu(x))+\int_{0}^{y}(d x /(\lambda(x)-\mu(x))) \leq 2 y / \underline{\delta}$. This means $y^{*} \geq K \underline{\delta}^{2} / 4 k_{1} d_{1}$.

Now with the help of (c1) and Proposition 3.1, (c2) can be proved in the same way as for (b) of Corollary 2.5. 


\section{Acknowledgments}

The authors are grateful to the reviewers for their valuable comments. This research is partially supported by the Alliance: Franco-British Research Partnership Programme, project "Impulsive Control with Delays and Application to Traffic Control in the Internet" (PN08.021). Y. Zhang thanks Mr. Daniel S. Morrison for his comments which improve the English presentation of this paper.

\section{References}

[1] O. Berman and D. Perry, "An EOQ model with state-dependent demand rate," European Journal of Operational Research, vol. 171, no. 1, pp. 255-272, 2006.

[2] R. Levin, C. McLaughlin, R. Lamone, and J. Kottas, Production/Operations Management: Contemporary Policy for Managing Operating Systems, McGraw-Hill, New York, NY, USA, 1972.

[3] T. L. Urban, "Inventory models with the demand rate dependent on stock and shortage levels," International Journal of Production Economics, vol. 40, no. 1, pp. 21-28, 1995.

[4] M. Ferguson, V. Jayaraman, and G. C. Souza, "Note: an application of the EOQ model with nonlinear holding cost to inventory management of perishables," European Journal of Operational Research, vol. 180, no. 1, pp. 485-490, 2007.

[5] B. C. Giri, S. Pal, A. Goswami, and K. S. Chaudhuri, "An inventory model for deteriorating items with stock-dependent demand rate," European Journal of Operational Research, vol. 95, no. 3, pp. 604610, 1996.

[6] B. C. Giri and K. S. Chaudhuri, "Deterministic models of perishable inventory with stock-dependent demand rate and nonlinear holding cost," European Journal of Operational Research, vol. 105, no. 3, pp. 467-474, 1998.

[7] H. J. Weiss, "Economic order quantity models with nonlinear holding costs," European Journal of Operational Research, vol. 9, no. 1, pp. 56-60, 1982.

[8] T. L. Urban, "Inventory models with inventory-level-dependent demand: a comprehensive review and unifying theory," European Journal of Operational Research, vol. 162, no. 3, pp. 792-804, 2005.

[9] N. Bäuerle, "Asymptotic optimality of tracking policies in stochastic networks," The Annals of Applied Probability, vol. 10, no. 4, pp. 1065-1083, 2000.

[10] A. Gajrat and A. Hordijk, "Fluid approximation of a controlled multiclass tandem network," Queueing Systems. Theory and Applications, vol. 35, no. 1-4, pp. 349-380, 2000.

[11] C. Maglaras, "Discrete-review policies for scheduling stochastic networks: trajectory tracking and fluid-scale asymptotic optimality," The Annals of Applied Probability, vol. 10, no. 3, pp. 897-929, 2000.

[12] G. Pang and M. V. Day, "Fluid limits of optimally controlled queueing networks," Journal of Applied Mathematics and Stochastic Analysis, Article ID 68958, 19 pages, 2007.

[13] A. Gajrat, A. Hordijk, and A. Ridder, "Large-deviations analysis of the fluid approximation for a controllable tandem queue," The Annals of Applied Probability, vol. 13, no. 4, pp. 1423-1448, 2003.

[14] A. Piunovskiy, "Controlled jump Markov processes with local transitions and their fluid approximation," WSEAS Transactions on Systems and Control, vol. 4, no. 8, pp. 399-412, 2009.

[15] A. B. Piunovskiy, "Random walk, birth-and-death process and their fluid approximations: absorbing case," Mathematical Methods of Operations Research, vol. 70, no. 2, pp. 285-312, 2009.

[16] H. E. Qi-Ming and E. M. Jewkes, "Performance measures of a make-to-order inventory-production system," IIE Transactions, vol. 32, no. 5, pp. 409-419, 2000.

[17] P. Köchel, "On queueing models for some multi-location problems," International Journal of Production Economics, vol. 45, no. 1-3, pp. 429-433, 1996.

[18] Y. Xu and X. Chao, "Dynamic pricing and inventory control for a production system with average profit criterion," Probability in the Engineering and Informational Sciences, vol. 23, no. 3, pp. 489-513, 2009.

[19] Y. U. S. Zheng and P. Zipkin, "Queueing model to analyze the value of centralized inventory information," Operations Research, vol. 38, no. 2, pp. 296-307, 1990.

[20] R. C. Baker and T. L. Urban, "A deterministic inventory system with an inventory-level-dependent demand rate," Journal of the Operations Research Society, vol. 50, no. 3, pp. 249-256, 1991. 
[21] T. K. Datta and A. K. Pal, "Note on an inventory model with inventory-level-dependent demand rate," Journal of the Operational Research Society, vol. 41, no. 10, pp. 971-975, 1990.

[22] T. Urban, "Inventory model with an inventory-level-dependent demand rate and relaxed terminal conditions," Journal of the Operational Research Society, vol. 43, no. 7, pp. 721-724, 1992.

[23] A. Piunovskiy and Y. Zhang, "Accuracy of fluid approximations tocontrolled birth-and-death processes: absorbing case," Mathematical Methods of Operations Research. In press.

[24] S. Ross, Introduction to Probability Models, Academic Press, New York, NY, USA, 2002.

[25] Q. Hu and J. Liu, An Introduction to Markov Decision Processes, Xidian University Press, Xi'an, China, 1999.

[26] Z. Wang and X. Yang, Birth-and-Death Processes and Markov Chains, Science press, Beijing, China, 2005. 


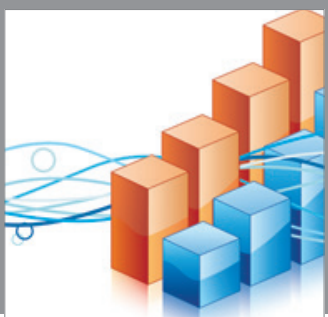

Advances in

Operations Research

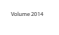

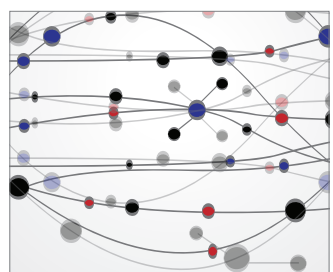

\section{The Scientific} World Journal
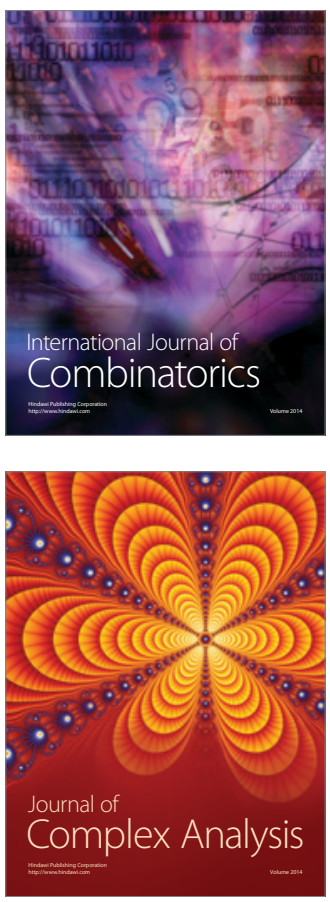

International Journal of

Mathematics and

Mathematical

Sciences
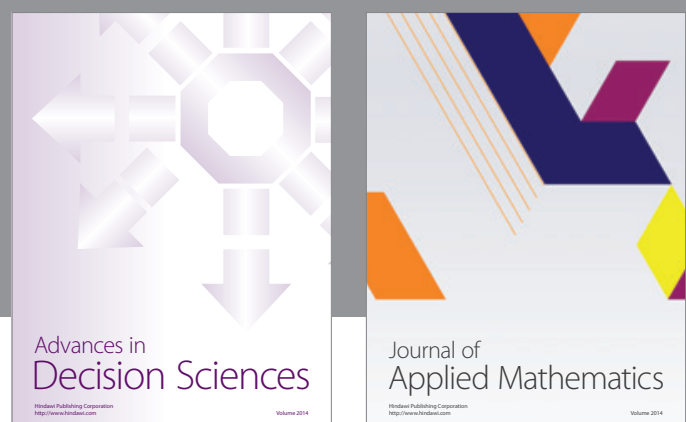

Journal of

Applied Mathematics
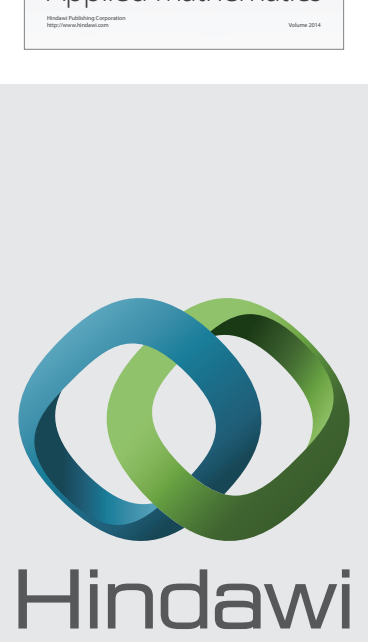

Submit your manuscripts at http://www.hindawi.com
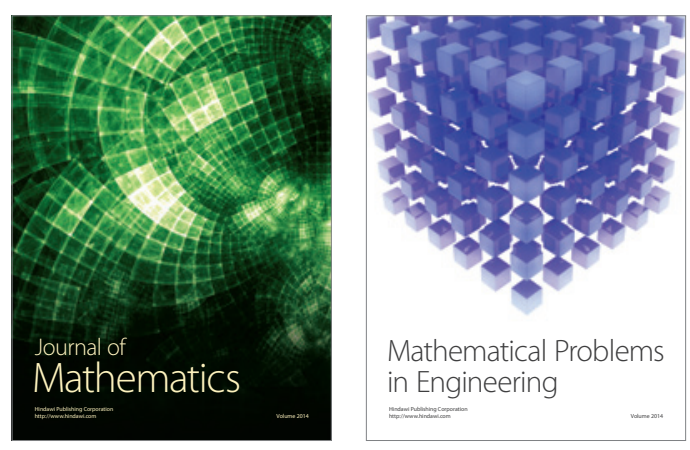

Mathematical Problems in Engineering
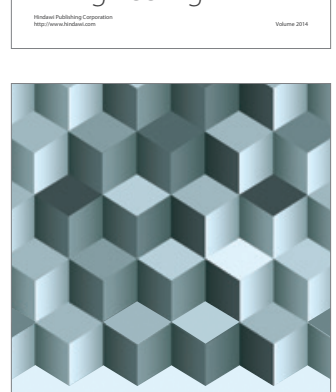

Journal of

Function Spaces
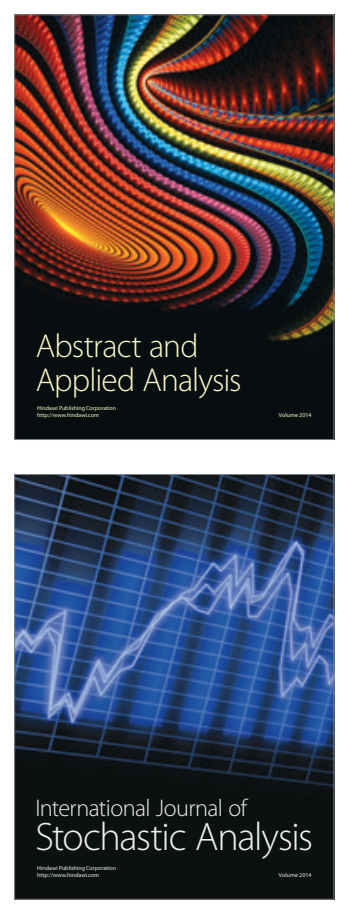

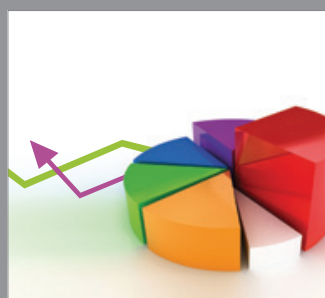

ournal of

Probability and Statistics

Promensencen
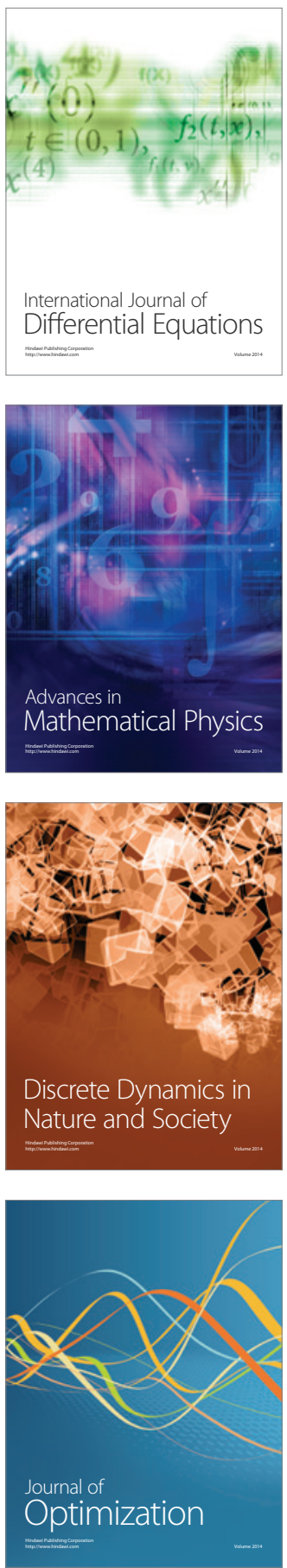